\title{
Dynamic model for energy security level assessment
}

\author{
J. Augutis ${ }^{1,2}$, S. Pečiulyté ${ }^{2}$, R. Krikštolaitis ${ }^{1,2}$, I. Žutautaite $\dot{e}^{1,2}$ \\ \& E. Ušpuras ${ }^{1}$ \\ ${ }^{1}$ Lithuanian Energy Institute, Lithuania \\ ${ }^{2}$ Vytautas Magnus University, Lithuania
}

\begin{abstract}
Effective functioning of the energy sector is very important for the economy of every modern state. Thus, energy security is an inseparable part of the total economic security of the state. Energy security indicators were constructed for the investigation of the Lithuanian energy security level. The purpose of the paper is to construct a dynamic model for energy security level assessment. Dynamic model of indicators will enable us to forecast the Lithuanian energy security level according to different factors, such as building of liquefied natural gas (LNG) terminal, power interconnections between Lithuania and Poland (LitPolLink), etc. Since technical parameters of new objects are not exactly known, these uncertainties should be reflected in the initial conditions of a differential equations system. Thus, in such conditions, the values of indicators are random variables. The paper presents a model of dynamic indicators and pilot calculations.
\end{abstract}

Keywords: energy supply security, dynamic model, energy security indicators.

\section{Introduction}

There are different interpretations of energy security conception. For instance, Yergin summarized energy security conception in the following way: "in the developed world the usual definition of energy security is simply the availability of sufficient supplies at affordable prices, different countries interpret what the concept means for them differently" [1].

To find rational solutions for energy supply security means to assess the costs and probability of supply disruptions, to compare the present energy supply 
security level with the forecasts, and to decide how to improve it. Different approaches are used for assessing security of energy supply: geopolitical assessment of scenarios, economical modelling, expert risk assessment, analysis of primary energy sources, and development of security indicators system [2]. A security indicator is a special index which provides numerical values to important issues for the security of energy sector. The values of security indicators may be obtained from statistical data. These values of indicators are usually obtained in the particular time moments, however, indicators change continuously. So, it is advisable to construct a dynamic model of indicators, which includes interdependencies between these indicators. The dynamic models of two types were shortly investigated in [3].

Dynamic model of indicators will enable us to forecast the Lithuanian energy security level according to different factors, such as building of liquefied natural gas (LNG) terminal, power interconnections between Lithuania and Poland (LitPolLink), etc. But in this case we should correct the initial conditions of differential equations system. Since technical parameters of new objects are not exactly known, these uncertainties should be reflected in the initial conditions. Thus, in such conditions, the values of indicators are random variables. Their estimates are obtained in this paper using a modified application of Bayesian approach.

\section{Dynamic indicator model}

Let us construct a homogeneous differential equations system, according to the interdependencies between indicators

$$
\left\{\begin{array}{l}
\frac{\mathrm{d} I_{1}(\mathrm{t})}{\mathrm{d} t}=a_{11} I_{1}(t)+a_{12} I_{2}(t)+a_{13} I_{3}(t) ; \\
\frac{\mathrm{d} I_{2}(\mathrm{t})}{\mathrm{d} t}=a_{21} I_{1}(t)+a_{22} I_{2}(t)+a_{23} I_{3}(t) ; \\
\frac{\mathrm{d} I_{3}(\mathrm{t})}{\mathrm{d} t}=a_{31} I_{1}(t)+a_{32} I_{2}(t)+a_{33} I_{3}(t) ;
\end{array}\right.
$$

here $a_{i j}, i, j=1,2,3$ are coefficients, $I_{i}, i=1,2,3$ - energy security indicators. Coefficients $a_{i j}, i, j=1,2,3$ may be calculated using different methods: algebraic, statistical, etc. In [5] we proposed to use correlations of indicators for calculation of these coefficients. In subsection 2.1 of this paper we describe algebraic method of coefficients calculation. Let us use the following definitions

$$
\mathrm{I}(t)=\left(\begin{array}{lll}
I_{1}(t) & I_{2}(t) & I_{3}(t)
\end{array}\right)^{\mathrm{T}}, A=\left(\begin{array}{lll}
a_{11} & a_{12} & a_{13} \\
a_{21} & a_{22} & a_{23} \\
a_{31} & a_{32} & a_{33}
\end{array}\right),
$$

then (1) differential equations system we can write in the matrix form

$$
\frac{\mathrm{dI}(t)}{\mathrm{d} t}=\mathrm{A} \mathrm{I}(t),
$$


Solution of differential equations system (2) may be found according to the formula (3)

$$
\mathrm{I}(t)=C_{1} B_{1} e^{\lambda_{1} t}+C_{2} B_{2} e^{\lambda_{2} t}+C_{3} B_{3} e^{\lambda_{3} t},
$$

here $\mathrm{B}=\left(b_{1}, b_{2}, b_{3}\right)^{\mathrm{T}}-$ vector of constants, $\lambda$ - eigenvalues of matrix $\mathrm{A}$.

\subsection{Coefficients for differential equations system of indicators}

Let us give the variation for each indicator $\Delta I_{i}(t)=\left(I_{i}(t+\Delta t)-I_{i}(t)\right) / \Delta t$, $i=1,2,3$ and construct system of algebraic equations

$$
\left\{\begin{array}{l}
\Delta I_{1}=a_{11} I_{1}(t)+a_{12} I_{2}(t)+a_{13} I_{3}(t) \\
\Delta I_{2}=a_{21} I_{1}(t)+a_{22} I_{2}(t)+a_{23} I_{3}(t) \\
\Delta I_{3}=a_{31} I_{1}(t)+a_{32} I_{2}(t)+a_{33} I_{3}(t)
\end{array}\right.
$$

here $a_{i j}, i, j=1,2,3$ are unknown coefficients, $I_{i}(t), i=1,2,3$ - energy security indicators. We take the values of each indicator in time moments $t_{i}, i=0, \ldots, 3$ and define $I_{i j}=I_{i}\left(t_{j}\right), i, j=1,2,3$. So, we have algebraic system of nine equations with nine unknowns

$$
\begin{aligned}
& \Delta I_{1 i}=a_{11} I_{1 i}+a_{12} I_{2 i}+a_{13} I_{3 i}, \\
& \Delta I_{2 i}=a_{21} I_{1 i}+a_{22} I_{2 i}+a_{23} I_{3 i}, \quad i=1, \ldots, 3 . \\
& \Delta I_{3 i}=a_{31} I_{1 i}+a_{32} I_{2 i}+a_{33} I_{3 i},
\end{aligned}
$$

We define vectors $\Delta I^{i}=\left(\Delta I_{i 1}, \Delta I_{i 2}, \Delta I_{i 3}\right)$ and $I^{i}=\left(I_{i 1}, I_{i 2}, I_{i 3}\right), i, j=1,2,3$. In this case the system of algebraic equations can be written in matrix form

$$
\Delta \mathrm{I}=\mathrm{AI} \text {, }
$$

here matrix of variations $\Delta \mathrm{I}$, matrix of coefficients $\mathrm{A}$ and matrix of indicators $\mathrm{I}$

$$
\Delta \mathrm{I}=\left(\begin{array}{lll}
\Delta \mathrm{I}_{11} & \Delta \mathrm{I}_{12} & \Delta \mathrm{I}_{13} \\
\Delta \mathrm{I}_{21} & \Delta \mathrm{I}_{22} & \Delta \mathrm{I}_{23} \\
\Delta \mathrm{I}_{31} & \Delta \mathrm{I}_{32} & \Delta \mathrm{I}_{33}
\end{array}\right), \quad \mathrm{A}=\left(\begin{array}{lll}
a_{11} & a_{12} & a_{13} \\
a_{21} & a_{22} & a_{23} \\
a_{31} & a_{32} & a_{33}
\end{array}\right), \mathrm{I}=\left(\begin{array}{lll}
I_{11} & I_{12} & I_{13} \\
I_{21} & I_{22} & I_{23} \\
I_{31} & I_{32} & I_{33}
\end{array}\right) .
$$

We get unknown coefficients of differential equations system from formula (6)

$$
\mathrm{A}=\Delta \mathrm{II}^{-1} \text {. }
$$

\subsection{Stochastic initial conditions of differential equations system}

Aiming to find a particular solution for differential equations system (2), i.e. particular expression of each indicator as on time dependent function, we need to formulate initial conditions for differential equations system. Constants $C_{i}$, $i=1,2,3$ of general solution may be found by solving system of differential equations (2) in time moment $t_{0}$

$$
I_{1}\left(t_{0}\right)=I_{1}^{0}, \quad I_{2}\left(t_{0}\right)=I_{2}^{0}, \quad I_{3}\left(t_{0}\right)=I_{3}^{0},
$$

here $I_{i}^{0}, i=1,2,3$ - normalized factual value of indicator in year $t_{0}$. 
Dynamic indicators model will enable us to forecast the Lithuanian energy security level according to different factors, such as building liquefied natural gas terminal, electricity connections with Sweden and Poland, etc. In this case we should correct the initial conditions (8) of differential equations system. Since technical parameters of new objects are not exactly known, these uncertainties should be reflected in the initial conditions. Thus, in such conditions, the values of indicators are random variables.

$$
I_{1}\left(t_{0}\right)=Y_{1}, \quad I_{2}\left(t_{0}\right)=Y_{2}, \quad I_{3}\left(t_{0}\right)=Y_{3},
$$

here $Y_{i}, i=1,2,3-$ a random variable. Since time of building of new objects is not exactly known, $t_{0}$ also may be a random variable.

Appropriate probability distributions of random variables $Y_{i}$ (at time $t$ ) are obtained performing Kolmogorov-Smirnov, Anderson-Darling or $\chi^{2}$ goodnessof-fit test. Note $p_{i}(y)$ is probability density function (pdf) of random variables $Y_{i}$. In general case $Y_{i}(t)$ is stochastic process with mean

$$
\mathrm{E} Y_{k}=\mathrm{E} I_{k}(t)=C_{1} b_{k 1} e^{\lambda_{1} t}+C_{2} b_{k 2} e^{\lambda_{2} t}+C_{3} b_{k 3} e^{\lambda_{3} t}, k=1,2,3,
$$

$b_{k i}$ and $\lambda_{i}$ are known constants, $C_{i}, i=1,2,3$ are independent random variables with pdfs $p_{i}\left(x_{i}\right), i=1,2,3$, or multidimensional pdf

$$
\varphi\left(x_{1}, x_{2}, x_{3}\right)=\prod_{i=1}^{3} p_{i}\left(x_{i}\right) .
$$

Methodology for the calculation of $C_{i}$ estimates has been based on the obtained values of indicators $Y_{i}$ but not on values of $C_{i}$ (that can be measured). Information on the dependency of indicators from each other (defined by equalities (3) or (10)) and pdfs of random variables $C_{i}$ have been taken into account as well. In mathematical statistics theory, the well known Bayesian approach (BA) allows a combination of two kinds of information: prior (for instance, generic statistic data of similar devices exploitation, subjective option of experts) and measurements or observations [3]. In case of unknown probability density functions of random variables $C_{i}$ non-informative, for instance, a uniform distribution or Jeffreys distribution [7] can be used as prior. In this research work non-stationary processes (NP) are analysed, i.e. the values of indicators depends on time $t$. Classical application of BM is not correct in this case, because observations obtained in different time moments represent the other state of the indicators. The modified application of BA for the calculation estimates of parameters of non-stationary process mathematical models is presented in research paper [8].

\section{Calculating example}

For the investigation of Lithuanian energy security level it is important to construct the model which includes interdependencies between indicators. This model will enable us to forecast the Lithuanian energy security level according to different factors, such as building of LNG terminal, Visaginas nuclear power 
plant (NPP), LitPolLink, etc. Thus, it is advisable to construct a dynamical indicators model for energy security level assessment.

As an example, three indicators are used for the analysis. The first one is technical indicator $\left(I_{1}(t)\right)$, which integrates technical parameters of energy system (electricity, gas, oil and heat). The main component of this indicator is installed power capacity of generators to maximal power demand in all mentioned sectors. Also this indicator joins energy system objects' lifetime, storage possibilities of resources, etc. The second indicator is an economic indicator $\left(I_{2}(t)\right)$, which integrates economic aspects of energy system functioning. This indicator is mostly related with market and prices in appropriate energy sectors, energy resources import, possibilities to choose suppliers, etc. And the last indicator is socio-political $\left(I_{3}(t)\right)$, which integrates geopolitical and socio-political aspects. It joins such things as political risk of resource supplier country and resource transit country, average expense for energy, degrees of undertaking the EU commitment, etc. The values of these indicators from 2001 till 2010 are used for the calculations. Normalized factual values of indicators, variations of indicators are written in matrixes $\Delta \mathrm{I}$ and $\mathrm{I}$, whereas matrix A is calculated according to formula (7).

Using formula (1) a differential equations system is constructed

$$
\left\{\begin{array}{l}
\frac{\mathrm{d} I_{1}(t)}{\mathrm{d} t}=0.069 I_{1}(t)-0.199 I_{2}(t)+0.063 I_{3}(t) \\
\frac{\mathrm{d} I_{2}(t)}{\mathrm{d} t}=-0.046 I_{1}(t)+0.127 I_{2}(t)-0.024 I_{3}(t) \\
\frac{\mathrm{d} I_{3}(t)}{\mathrm{d} t}=0.258 I_{1}(t)-0.73 I_{2}(t)+0.458 I_{3}(t)
\end{array}\right.
$$

General solution of (12) differential equation is written in formula (13)

$$
\left\{\begin{array}{c}
I_{1}(t)=-0.158 C_{1} \mathrm{e}^{0.553 t}+0.943 C_{2} \mathrm{e}^{-0.002 t}-0.292 C_{3} \mathrm{e}^{0.103 t} \\
I_{2}(t)=0.072 C_{1} \mathrm{e}^{0.553 t}+0.334 C_{2} \mathrm{e}^{-0.002 t}+0.333 C_{3} \mathrm{e}^{0.103 t} \\
I_{3}(t)=-0.985 C_{1} \mathrm{e}^{0.553 t}-0.0002 C_{2} \mathrm{e}^{-0.002 t}+0.897 C_{3} \mathrm{e}^{0.103 t}
\end{array}\right.
$$

Constants $C_{i}, \quad i=1,2,3$ of general solution (13) are calculated using normalized factual values of indictors $I_{i}, i=1,2,3$ in 2010: $C_{1}=10.911, C_{2}=$ 27.187, $C_{3}=73.916$. Aiming at valuating Lithuanian energy security state after building LNG terminal in 2014 or LitPolLink in 2015, we need to correct values of obtained constants according to forecasted values of indicators.

Information about LNG terminal or LitPolLink start-up date, power and costs are inaccurate. In general case, values of indicators are unknown, i.e. assumed as random variables. Generalized information is used to forecast values of indicators and errors of estimates. Probable values $I_{i}{ }^{*}$ of indicators $I_{i}$ in cases of three different scenarios are presented in table 1. In this research paper four scenarios are analysed: scenario 2 - LNG plant will start-up in 2014; scenario 3 - LitPolLink will be operational in 2015; scenario 4 - LNG starts-up in 2014 and LitPolLink starts operate in 2015; scenario 1 means that neither LNG nor LitPolLink are constructed. 
Table 1: $\quad$ Probable values of indicators in cases of three different scenarios.

\begin{tabular}{|l|c|c|c|}
\hline \multicolumn{1}{|c|}{ Indicator } & Technical & Economic & Socio-political \\
\hline Scenario & 99.61 & 73.92 & 51.24 \\
\hline Scenario 2 & 99.51 & 71.71 & 51.48 \\
\hline Scenario 3 & 104.32 & 68.29 & 51.48 \\
\hline
\end{tabular}

Let's assume that at time moment $t$ probability distributions of indicators $I_{k}(t)$ are normal with means that satisfy equality (10).

$$
\mathrm{E} Y_{k}=\mathrm{EI}_{k}(t)=C_{1} b_{k 1} e^{\lambda_{1} t}+C_{2} b_{k 2} e^{\lambda_{2} t}+C_{3} b_{k 3} e^{\lambda_{3} t}, \quad k=1,2,3,
$$

$b_{k i}$ and $\lambda_{i}$ are known constants, $\sigma_{k}-$ standard deviations, $C_{i}(i=1,2,3)$ are independent random variables. $C_{i}$ can be determined using sum of constants and indicators products (form system of equations (14)). In case of normal distributions of indicators, probability distributions of random variables $C_{i}$ $(i=1,2,3)$ are normal as well with means equal to values that are calculated for 2010 , i.e. general solution of system of equations (14). Denote means are $c_{1}=10.911, c_{2}=27.187, c_{3}=73.916$ and standard deviations are $10 \%$ of its means, i.e. $s_{i}=0.1 c_{i}(i=1,2,3)$. Multidimensional probability density function

$$
\varphi\left(x_{1}, x_{2}, x_{3}\right)=\prod_{i=1}^{3} \frac{1}{s_{i} \sqrt{2 \pi}} \exp \left(-\frac{1}{2 s_{i}^{2}}\left(x_{i}-c_{i}\right)^{2}\right) .
$$

Values of indicators are used to update pdf of random variables $C_{i}$ ( $i=$ 1,2,3), by modified application of BA

$$
\begin{aligned}
& \varphi\left(x_{1}, x_{2}, x_{3} \mid I_{1}^{*}, I_{2}^{*}, I_{3}^{*}\right)= \\
& =\frac{\varphi\left(x_{1}, x_{2}, x_{3}\right) L\left(I_{1}^{*}, I_{2}^{*}, I_{3}^{*} \mid x_{1}, x_{2}, x_{3}\right)}{\int_{-\infty}^{\infty} \int_{-\infty}^{\infty} \int_{-\infty}^{\infty} \varphi\left(u_{1}, u_{2}, u_{3}\right) \mathrm{L}\left(I_{1}^{*}, I_{2}^{*}, I_{3}^{*} \mid u_{1}, u_{2}, u_{3}\right) \mathrm{d} u_{1} \mathrm{~d} u_{2} \mathrm{~d} u_{3}},
\end{aligned}
$$

here likelihood function

$$
\mathrm{L}\left(I_{1}^{*}, I_{2}^{*}, I_{3}^{*} \mid x_{1}, x_{2}, x_{3}\right)=\prod_{i=1}^{3} \exp \left(-\frac{1}{2 \sigma_{\mathrm{i}}^{2}}\left(\mathrm{I}_{\mathrm{i}}^{*}-\sum_{\mathrm{l}=1}^{3} \mathrm{x}_{\mathrm{l}} \mathrm{b}_{\mathrm{il}} \mathrm{e}^{\lambda_{1} \mathrm{t}^{*}}\right)^{2}\right) .
$$

Updated point estimates of random variables $C_{i}, i=1,2,3$, are

$$
\hat{C}_{i}=\int_{-\infty}^{\infty} \int_{-\infty}^{\infty} \int_{-\infty}^{\infty} \varphi\left(x_{1}, x_{2}, x_{3} \mid I_{1}^{*}, I_{2}^{*}, I_{3}^{*}\right) \mathrm{d} x_{1} \mathrm{~d} x_{2} \mathrm{~d} x_{3}, i=1,2,3 .
$$

Calculated point estimates and standard deviations of random variables $C_{i}$ $(i=1,2,3)$, are presented in table 2 . 
Table 2: $\quad$ Estimates of $C_{i}(i=1,2,3)$ obtained by BA of different scenarios.

\begin{tabular}{|l|c|c|c|c|c|c|}
\hline \multicolumn{1}{|c|}{ Constant } & \multicolumn{2}{|c|}{$\boldsymbol{C}_{\mathbf{1}}$} & \multicolumn{2}{c|}{$\boldsymbol{C}_{\mathbf{2}}$} & \multicolumn{2}{c|}{$\boldsymbol{C}_{\mathbf{3}}$} \\
\hline Scenario & Estimate & $\begin{array}{c}\text { Stand. } \\
\text { deviation }\end{array}$ & Estimate & $\begin{array}{c}\text { Stand. } \\
\text { deviation }\end{array}$ & Estimate & $\begin{array}{c}\text { Stand. } \\
\text { deviation }\end{array}$ \\
\hline Scenario 2 & 10.93 & 0.71 & 134.59 & 2.49 & 71.99 & 3.32 \\
\hline Scenario 3 & 10.63 & 0.61 & 135.38 & 3.22 & 72.33 & 3.55 \\
\hline Scenario 4 & 10.38 & 0.60 & 138.76 & 2.54 & 70.99 & 3.46 \\
\hline
\end{tabular}

In figs 1-3 are given functions of technical, economic and socio-political indicators in scenarios $1-4$.

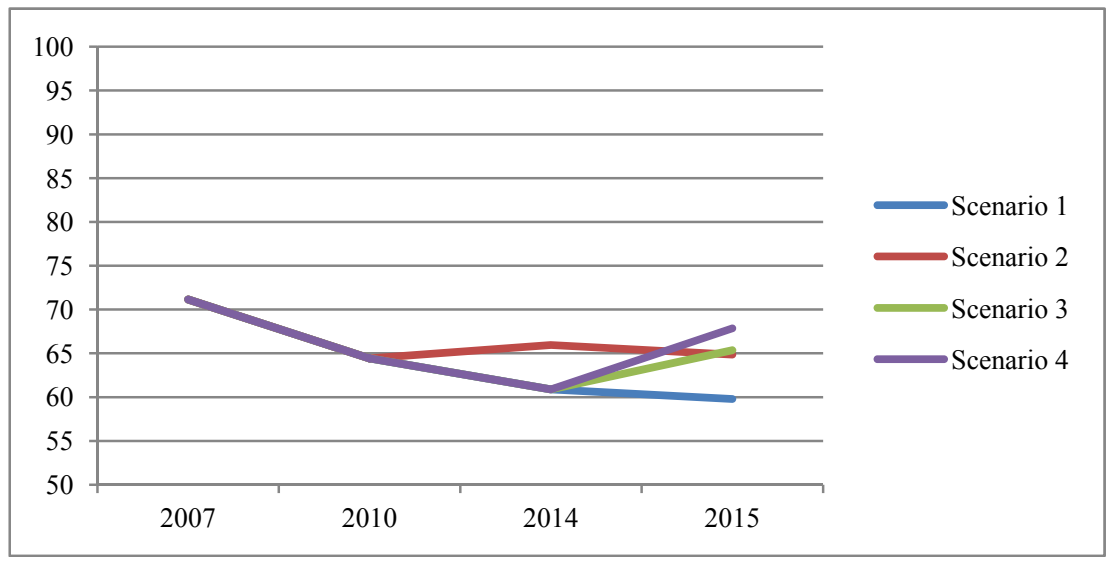

Figure 1: $\quad$ Graph of technical indicator $I_{1}(t)$.

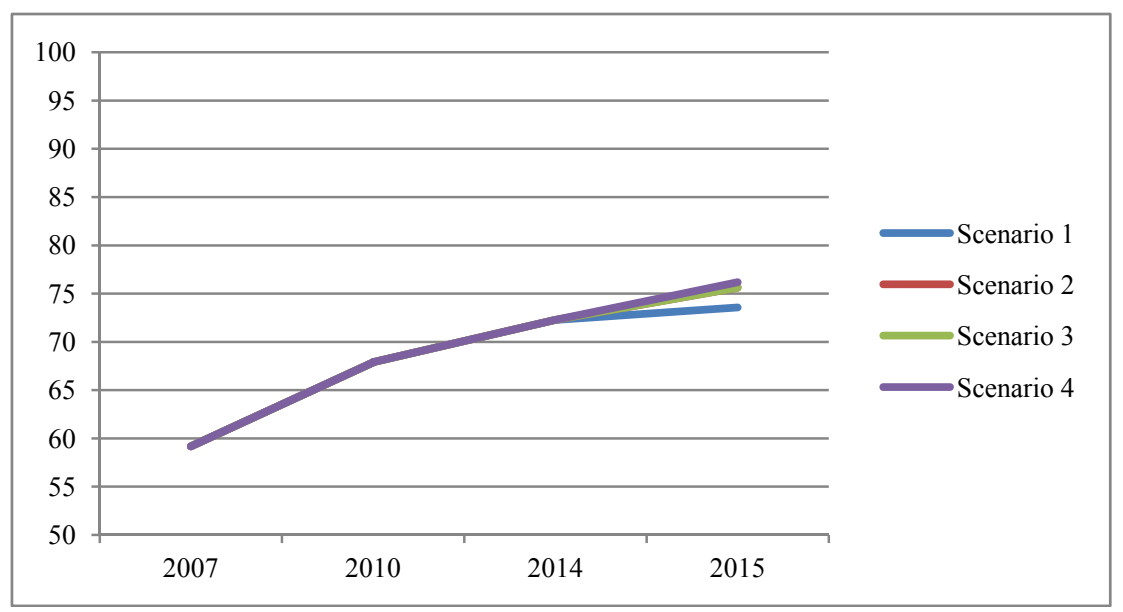

Figure 2: $\quad$ Graph of economic indicator $I_{2}(t)$. 


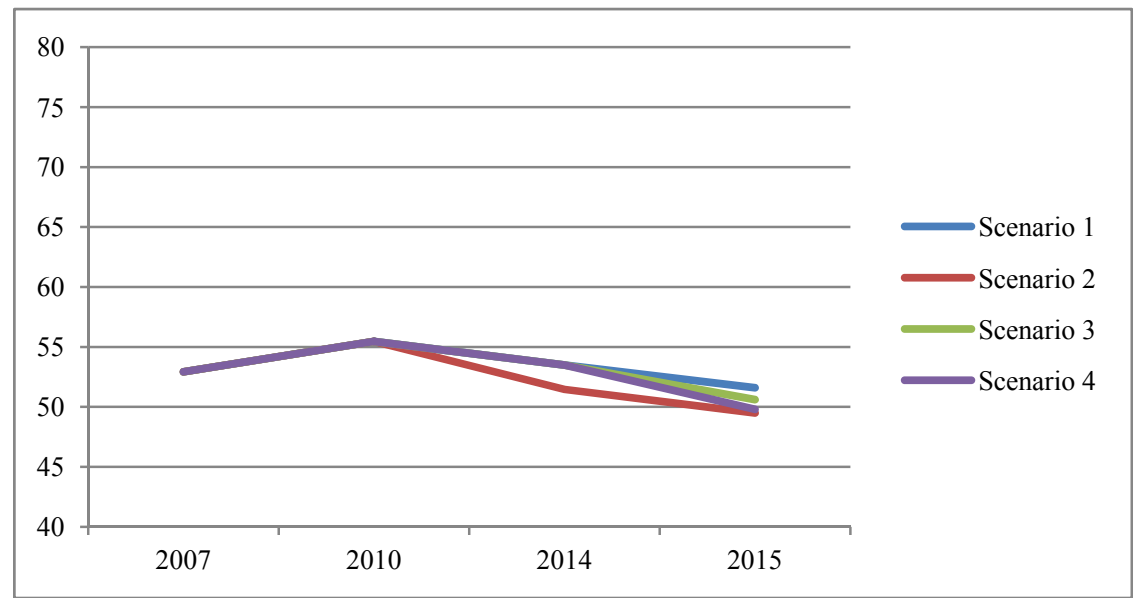

Figure 3: $\quad$ Graph of socio-political indicator $I_{3}(t)$.

Analyzing change of indicator security points, several things may be observed. Firstly, security level of technical indicator diminishes. This is related with the fact that Ignalina NPP units were shut down and at the same time generation capacities had changed. Besides, after shutdown of Ignalina NPP, larger amount of gas had to be used for electricity production. Speaking about economic indicator, the appearance of free electricity market and import of cheaper electricity had influence on its increase. Implementation of corresponding EU directives and improvement of geopolitical risk of respective state energy suppliers and transitional countries had a positive influence on the dynamics of sociopolitical indicator.

In order to identify the energy security state of the system, a 15-point system assessment scale is used. The scale is divided into three main parts: normal state (11-15 points), pre-critical state (6-10 points) and critical state (1-5 points). These three parts are further subdivided into five equal parts accordingly. The value of each indicator is evaluated by points from 1 to 15 during the research year. The state of energy supply security is evaluated taking into account the weights of indicators (we used equal weights of indicators) and the evaluation of indicators according to the following formula

$$
B=s_{1} I_{1}^{p}+s_{2} I_{2}^{p}+s_{3} I_{3}^{p}
$$

here $I_{i}^{p}, i=1,2,3$ - value of indicator in points, $s_{i}, i=1,2,3$ - weight of each indicator. Integral characteristics of Lithuanian energy supply security is presented in table 3 and fig. 4 for scenarios 1-4.

Statistical data of 2004-2011 was used for the verification of the proposed method. Constants of the model were calculated using data of 2004-2010; values of indicators and energy supply security state were calculated for 2011. The mean error of results (equals to 0.21) was calculated by comparing obtained values with actual values of indicators of 2011 . 
Table 3: $\quad$ Lithuanian energy supply security state.

\begin{tabular}{|c|c|c|c|}
\hline Year & & & \\
\hline Scenario & 2010 & 2014 & 2015 \\
\hline Scenario 1 & 8.67 & 9.00 & 8.67 \\
\hline Scenario 2 & 8.67 & 9.33 & 9.33 \\
\hline Scenario 3 & 8.67 & 9.00 & 9.33 \\
\hline Scenario 4 & 8.67 & 9.33 & 9.67 \\
\hline
\end{tabular}

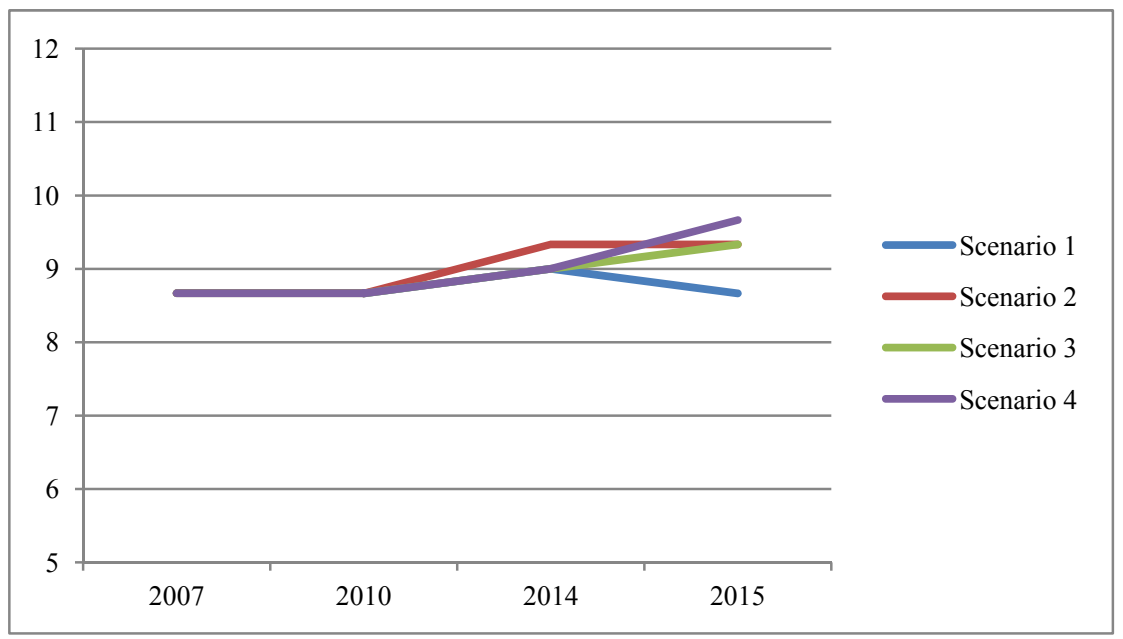

Figure 4: $\quad$ Lithuanian energy supply security state.

\section{Conclusions}

1. The created method of energy supply security assessment is appropriate and valid. The difference between the described method and the best estimate method is small. The created method enables us to evaluate data uncertainty.

2. The proposed method allows us to estimate the uncertainties of characteristics of energy development projects as most of this data (finish of the object construction, cost of the object, etc.) is random variables.

3. The created methodology enables us to compare integral characteristics of energy supply security for different scenarios.

4. According to this methodology, the energy security level is the highest (9.67 points in a 15-point system) after building LNG terminal and electricity interconnection between Lithuania and Poland in 2015. However, the aggregation of all positive and negative consequences leads to the conclusion that energy security level did not significantly change in all scenarios. In all cases the energy security level is seen as pre-critical. 
5. In pessimistic scenario, when no new energetics objects are built, Lithuanian energy supply security level decreases to pre-critical level to 8.67 points in 2015 .

\section{Acknowledgement}

This research was funded by Grants (No. ATE-06/2012 and No. ATE-04/2012) from the Research Council of Lithuania.

\section{References}

[1] Yergin, D., Energy security in the 1990s. Foreign Affairs, 67(1), pp. 111, 1988.

[2] Augutis, J., Krikštolaitis, R., Pečiulytè, S., Konstantinavičiūtė, I., Sustainable development and energy security level after Ignalina NPP shutdown. Technological and Economic Development of Economy, 17(1), pp. 5-21, 2011.

[3] Bykova, E. V., Methods of calculation and analysis of energy security. Kishinev, pp. 156, 2005. (in Russian).

[4] Mixalevich, A.A., Bykova, E.V., Postolatii, V.M., Fisenko, S.P., Shnip, A.I., Rimko, D.V., Grodecki, M.V., Methodological approaches to solving the energy security problem in Moldova and Belarus. Kishinev, pp. 100, 2010. (in Russian).

[5] Augutis, J., Krikštolaitis, R., Pečiulytè, S., Lithuanian energy security level assessment based on indicator dependence . Safety and security engineering IV: [proceedings]: pp. 71-82, 2011.

[6] Bernardo, J. M., Smith, A. F. M., Bayesian theory. John Wiley \& Sons, pp. 357-361, 2003.

[7] Berthold, M., Hand, D.J., Intelligent Data Analysis. 2nd edition, pp. 138$139,2003$.

[8] Augutis, J., Žutautaite, I., Radziukynas, V., Krikštolaitis, R., Kadiša, S., Application of Bayesian method for electrical power system transient stability assessment. International journal of electrical power and energy systems, 42, pp. 465-472, 2012. 